\title{
Trigger Spray Dispensing Plastic Container Closure
}

National Cancer Institute

\section{Source}

National Cancer Institute. Trigger Spray Dispensing Plastic Container Closure. NCI

Thesaurus. Code C96136.

Closure designed to dispense product from containers by spraying the product when a trigger is pulled. 